\title{
Monodispersed Bioactive Glass Nanoparticles Enhance the Osteogenic Differentiation of Adipose-Derived Stem Cells through Activating TGF-Beta/Smad3 Signaling Pathway
}

\author{
Yi Guo, Yumeng Xue, Wen Niu, Mi Chen, Min Wang, Peter X. Ma, and Bo Lei*
}

It is important to understand the interaction mechanisms between nanomaterials and adipose-derived stem cells for biomedical application. Nanoscale bioactive glass has positive effects on guiding osteoblasts differentiation and bone regeneration. However, the effects and molecular mechanism of monodispersed bioactive glass nanoparticles on the osteogenic differentiation of adipose-derived stem cells are still not clear up to now. In this study, the effects and underlying molecular mechanism of monodispersed bioactive glass nanoparticles on the osteogenic differentiation of adipose-derived stem cells are investigated in minute detail. The results show that nanoparticles (100-200 nm) can be absorbed by stem cells and is distributed in cytoplasm and nucleus. In both culture conditions (normal and osteoinductive), nanoparticles $\left(80 \mu \mathrm{g} \mathrm{mL}^{-1}\right)$ can significantly enhance the osteogenic differentiation of stem cells through upregulating the alkaline phosphatase activity, osteogenic genes and protein expressions, as well as calcium deposition. Further study suggests that the activation of transforming growth factor-beta/Smad3 signaling pathway plays an important role in the osteogenic differentiation of adipose-derived stem cells enhanced by monodispersed nanoparticles. This study may have important implications for better understanding of stem cells fate induced by monodispersed nanoparticles and provide a promising approach toward stem cells-based bone regeneration.

\section{Introduction}

Mesenchymal stem cells (MSCs) are thought to be multipotent cells, which have the ability to differentiate into multiple cell types in vitro, including cartilage, adipose, muscle, bone, neuron, and liver. ${ }^{[1,2]}$ The cultivation and selective differentiation of MSC are important to tissue engineering. ${ }^{[3]}$ And the bone marrow MSC (BMSC) has been regarded as the gold standard of adult MSCs. ${ }^{[4]}$ However, the harvest of bone marrow is a relatively painful procedure and the yields of stem cells are also very low (bone marrow aspirate per milliliter contains only $0.001 \%$ to $0.01 \%$ stem cells), which may restrict their biomedical applications. ${ }^{[5]}$ To overcome these drawbacks for BMSCs, the adipose-derived stem cells (ASCs) have been proposed as an alternative stem cell population. ${ }^{[6]}$ In contrast to bone marrow, $1 \mathrm{~g}$ of adipose tissue includes $\approx 10 \%$ ASCs and the harvest is also obtained easily. ${ }^{[7,8]}$ Owing to the selfrenewal and regenerative capacity, ASCs have been induced to differentiate into desired cell types for applications in regenerative medicine and tissue engineering. ${ }^{[9]}$ As such, the ASC may be a more suitable stem cell than the bone marrow MSC for clinical tissue regeneration application.

Due to the increasingly aged population and traffic accident, bone tissue engineering has drawn much attention in recent years. ${ }^{[10,11]}$ Here, ASCs play an important role in enhancing bone tissue repair and regeneration. However, how to direct the osteogenic differentiation of ASCs efficiently has become the key factor for their successful application in bone tissue engineering. ${ }^{[12]}$ The osteogenic differentiation of ASCs could be induced by bioactive materials, osteogenic chemicals and growth factors. ${ }^{[13-15]}$ For example, D-glyceraldehyde3-phosphate, L-ascorbic acid, and dexamethasone have been used to induce the osteogenic differentiation of ASCs in vitro;
Y. Guo, Y. Xue, W. Niu, M. Chen, M. Wang, Prof. P. X. Ma, Prof. B. Lei

Frontier Institute of Science and Technology

Xi'an Jiaotong University

Xi'an 710054, China

E-mail: rayboo@xjtu.edu.cn

Prof. P. X. Ma

Department of Biologic and Materials Sciences

Department of Biomedical Engineering

Macromolecular Science and Engineering Center

Department of Materials Science and Engineering

University of Michigan

Ann Arbor, MI 48109, USA

\author{
Prof. B. Lei \\ State Key Laboratory for Mechanical Behavior of Materials \\ Xi'an Jiaotong University \\ Xi'an 710054, China \\ Prof. B. Lei \\ State Key Laboratory for Manufacturing Systems Engineering \\ Xi'an Jiaotong University \\ Xi'an 710054, China \\ Prof. B. Lei \\ Instrument Analysis Center \\ Xi'an Jiaotong University \\ Xi'an 710054, China
}

DOI: 10.1002/ppsc.201800087 
however, their safety in vivo was still not clear. ${ }^{[16]}$ The biological growth factors such as bone morphogenetic proteins (BMPs) were also employed to induce the osteogenic differentiation of ASCs, but they usually showed high cost and low specificity. ${ }^{[17]}$ Bioactive biomaterials such as bioactive glass (BG) possessed inherent enhanced osteogenic differentiation ability for BMSCs and osteoblasts, as well as reinforced bone tissue regeneration in vivo. ${ }^{[18-20]}$ However, previous studies showed that bioactive glass scaffolds have no significant effects on the osteogenic differentiation of ASCs. ${ }^{[21]}$

There are increasing pieces of evidence that nanoscale biomaterials could significantly enhance the osteogenic differentiation of MSC and tissue formation in vivo. ${ }^{[2,23]}$ For examples, gold nanoparticles, well-ordered $\mathrm{SrTiO}_{3}$ nanotube arrays, and amino acid-functionalized calcium phosphate nanoparticles could stimulate the osteogenic differentiation of MSCs. ${ }^{[24]}$ Different from the macroscale bioactive glass-based biomaterials, bioactive glass nanoparticles (BGNs) possess biomimetic micro-nanoscale topological structure, high surface area, enhanced biomineralization ability, and bone tissue regeneration activity. ${ }^{[25]}$ Monodispersed BGNs were also developed and have shown promising applications in bioimaging, drug/gene delivery. ${ }^{[26,27]}$ Monodispersed nanoscale particle size could be easily absorbed by cells and probably induce some positive differentiation behavior of cells. Previous studies also demonstrated that BGNs could efficiently improve the osteogenic differentiation of osteoblasts and BMSCs through a mitogenactivated protein kinases (MAPK) pathway. ${ }^{[2,29]}$ Although the effect of nanoscale biomaterials on stem cell fate is evident, the interactions and molecular mechanisms between biocompatible nanoparticles and ASCs are still not demonstrated in detail.

Herein, the effects of monodispersed BGNs on the osteogenic differentiation of ASCs and related molecular mechanism were studied. We investigated the effects of BGNs on the proliferation, alkaline phosphatase activity (ALP), osteogenic protein and genes expressions, and calcium mineralization of ASCs. Recent studies illustrated that both the Smad and p38 MAPK pathways in transforming growth factor (TGF)-beta play a critical role in Runx2 expression and corresponded osteogenic differentiation in mesenchymal precursor cell differentiation. ${ }^{[30,31]}$ And BGNs could significantly enhance the Runx2 expressions even under the normal growth medium. Therefore, we hypothesized that monodispersed BGNs may interact and internalize with ASCs, and activate the TGF-beta/Smad3 signaling pathway to induce ASCs osteogenic differentiation.

\section{Result and Discussion}

\subsection{Synthesis and Characterizations of Monodispersed BGNs}

The monodispersed BGNs were successfully synthesized by a typical sol-gel process. The structure characterizations of BGNs are shown in Figure S1 in the Supporting Information. The transmitted electron microscopy (TEM) images showed that BGNs possess a monodispersed spherical nanoscale size of 200-300 nm (Figure S1A, Supporting Information). The mean hydrodynamic diameter of BGNs was about $389 \mathrm{~nm}$ with a Zeta potential of $-12 \mathrm{mV}$ (Table S1, Supporting Information).
The energy dispersive spectra (EDS) demonstrated the typical elements (Si and $\mathrm{Ca}$ ) in BGNs (Figure S1B, Supporting Information). The Fourier transform infrared (FT-IR) spectra exhibited the characteristic bands of Si-O-Si at $1000-1100 \mathrm{~cm}^{-1}$ (Figure S1C, Supporting Information). The amorphous structure of BGNs was confirmed by the X-ray diffraction analysis (Figure S1D, Supporting Information). The synthesized monodispersed BGNs may have positive effect on the proliferation and osteogenic differentiation of ASCs.

\subsection{Cellular Toxicity and Cell Uptake of BGNs}

ASCs were used to analyze the cytotoxicity of BGNs. After incubating with BGNs $\left(0-240 \mu \mathrm{g} \mathrm{mL}^{-1}\right)$ for 1 and $5 \mathrm{~d}$, no dead cells (red) were observed, which suggested the low cytotoxicity of BGNs (Figure 1A). The ASCs viability in various groups was significantly increased with the incubation time (from 1 to $5 \mathrm{~d}$ ), indicating that BGNs with different concentrations could support the cell proliferation (Figure 1B). On day 1, the BGNs group with $60 \mu \mathrm{g} \mathrm{mL} \mathrm{mL}^{-1}$ showed the significantly high cell viability compared with other groups (Figure $1 \mathrm{~B}$ ). On day 5 , the cell viability was slightly decreased as increasing the particles' concentrations. These results suggested that BGNs with the concentrations below $100 \mu \mathrm{g} \mathrm{mL} \mathrm{m}^{-1}$ may be suitable to investigate their effect on the osteogenic differentiation of ASCs.

Previous studies have shown that the cellular uptake of monodispersed nanoparticles probably enhanced the osteogenic differentiation of MSCs through mechanical-responsive signal pathway. ${ }^{[32]}$ Therefore, here, we investigated the cellular uptake of BGNs in ASCs (Figure 2). After incubation with fluorescent BGNs $\left(100 \mu \mathrm{g} \mathrm{mL} \mathrm{m}^{-1}\right)$ for $24 \mathrm{~h}$, the bright green and red fluorescence from nanoparticles in ASCs could be observed. The confocal laser scanning microscope (CLSM) images with high magnification further showed that the nanoparticles were distributed in cytoplasm and nucleus, suggesting the efficient uptake by ASCs. The intracellular distribution of BGNs may have important effect on the osteogenic differentiation of ASCs through the interaction between nanoparticles and intracellular proteins.

\subsection{Osteogenic Differentiation of ASCs}

The osteogenic differentiation of pluripotent progenitor ASCs is a crucial step in osteogenesis. The ALP activity is an early phenotypic marker for osteogenic differentiation, and mineralized nodule formation is a phenotypic marker for last stage in mature osteoblasts. Figure 3 shows the effect of BGNs with different concentrations on the ALP activity of ASCs under normal and osteoinductive conditions. Under normal medium condition, the ALP activity of ASCs was increased significantly with the BGNs concentration (Figure 3A). Compared with other groups, BGNs at $80 \mu \mathrm{g} \mathrm{mL} \mathrm{m}^{-1}$ showed the significantly high ALP activity on day 7. Different from the normal condition, the ASCs in BGNs $80 \mu \mathrm{g} \mathrm{mL} \mathrm{m}^{-1}$ also have exhibited the highest ALP activity on day 7 , among all groups (Figure $3 \mathrm{~B}$ ). The results showed that BGNs with suitable concentration could significantly enhance the ALP activity of ASCs. In addition, 

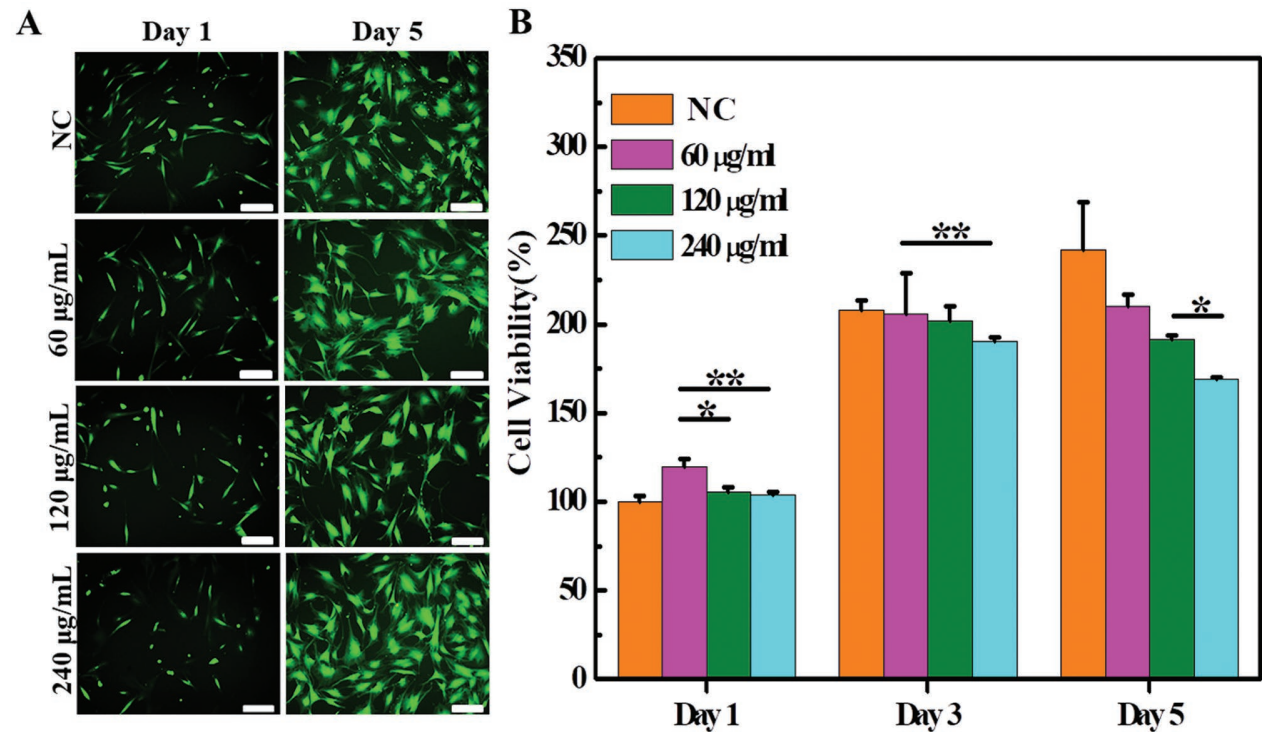

Figure 1. Cellular biocompatibility evaluation of ASCs following incubation with various concentration of BGNs. A) Fluorescence images indicating live-dead staining after day 1 and 5 incubation (scale bar: $100 \mu \mathrm{m}$ ). B) Cell viability and proliferation after culture for 1 to $5 \mathrm{~d}$. $* P<0.05$ and $* * P<0.01$.

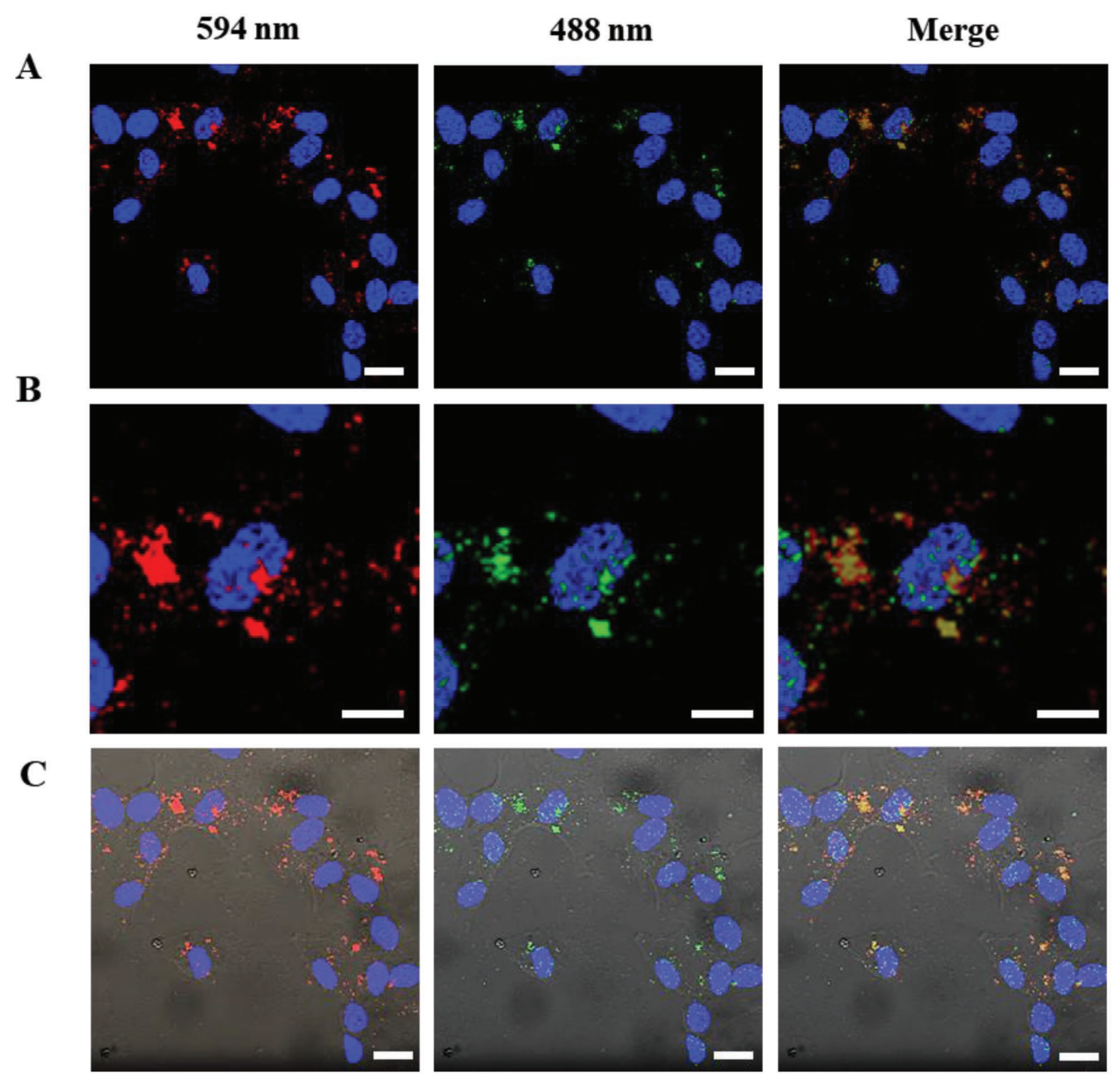

Figure 2. Confocal images of live ASCs after stained by fluorescent BGNs, the cell nuclei were stained as blue. A) Fluorescent images of cells after excitation at $594 \mathrm{~nm}$ (Red) and $488 \mathrm{~nm}$ (Green); B) Magnified fluorescent images of single cell showing the BGNs distribution; C) Merged images of fluorescent and bright field. Scale bar $=20 \mu \mathrm{m}$. 

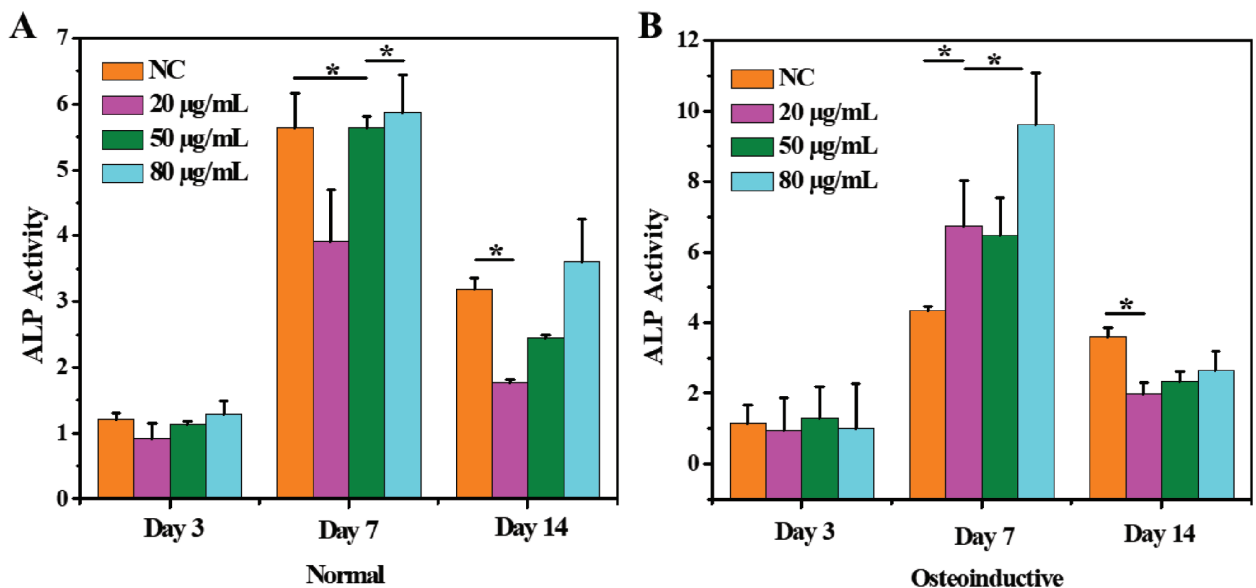

Figure 3. In vitro ALP activity evaluation of ASCs following induction by various concentrations of BGNs in normal and osteoinductive medium. A) ALP activity on days 3,7 , and 14 with normal culture medium; B) ALP activity on days 3,7 , and 14 with osteoinductive medium. $* P<0.05$ and $* * P<0.01$.

the Alizarin Red staining evaluation showed that BGNs could significantly improve the calcium mineralization of ASCs after $21 \mathrm{~d}$ (Figure 4). Under normal and osteoinductive condition, BGNs groups with various concentrations demonstrated the significantly high positive staining compared with tissue culture plate (NC) (Figure 4A). The relative intensity calculation further confirmed that BGNs groups have the significantly high positive staining either normal or osteoinductive condition (Figure 4B,C). These results suggested that BGNs could significantly enhance the calcium deposition and mineralization of ASCs.

The immunofluorescence staining for RUNX2and OPN proteins in ASCs on day 21 is shown in Figure 5. Under normal medium condition, there was no obvious positive RUNX2 and
$\mathbf{A}$
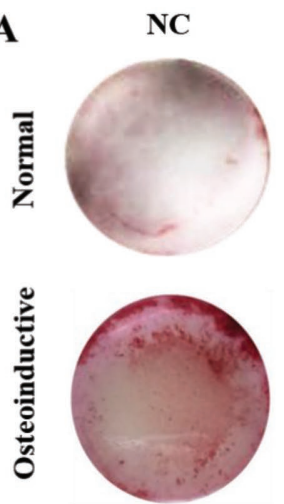

B

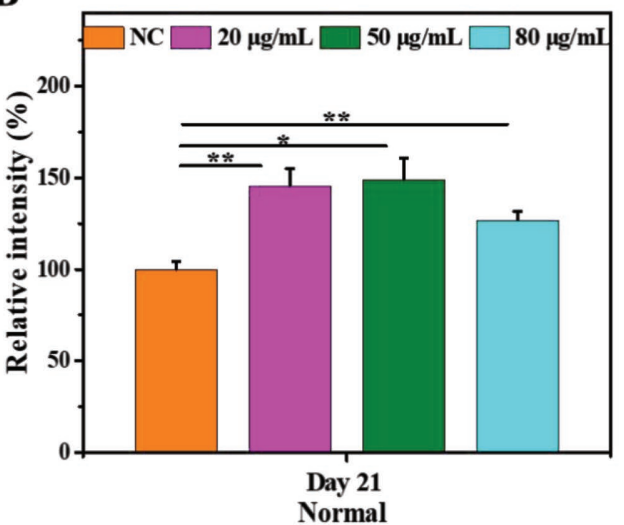

$20 \mu \mathrm{g} / \mathrm{mL}$
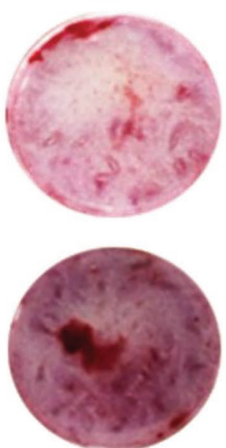

C

$50 \mu \mathrm{g} / \mathrm{mL}$
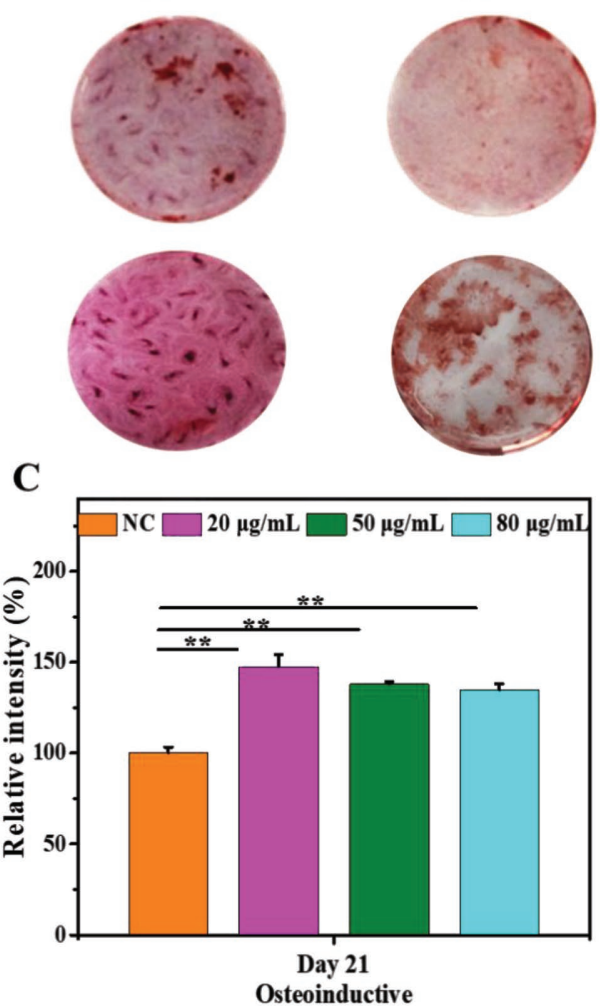

Figure 4. Calcium deposition biomineralization staining evaluation of ASCs after incubated with various concentrations of BGNs in normal and osteoinductive medium for $21 \mathrm{~d}$. A) Alizarin red staining analysis demonstrating the effect of BGNs on mineralized nodule of ASCs; B,C) The relative gray intensity based on the images of Alizarin red staining images analyzed by image J. Tissue culture plate (NC) is the negative control. $* P<0.05$ and $* * P<0.01$. 
A
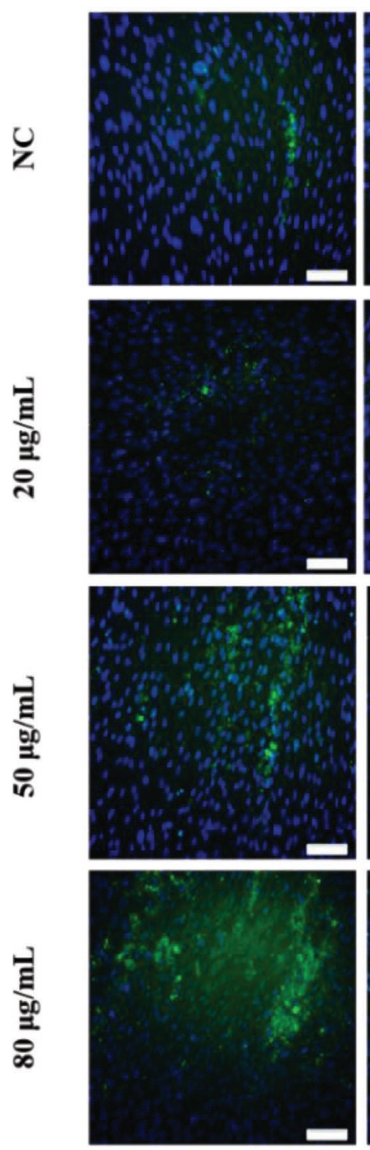
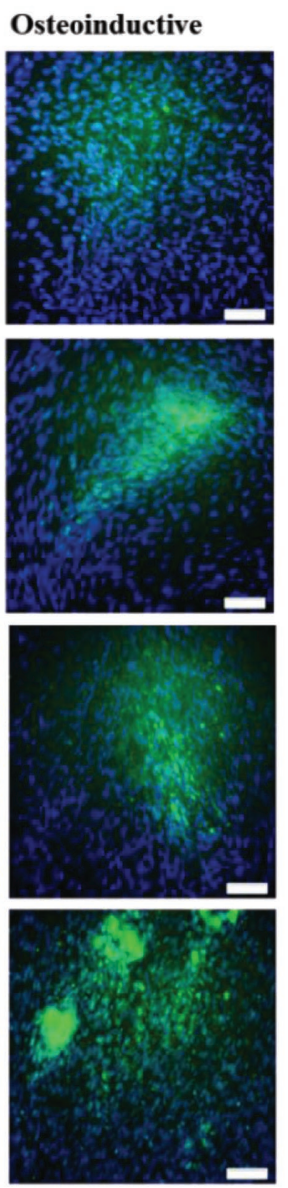

B
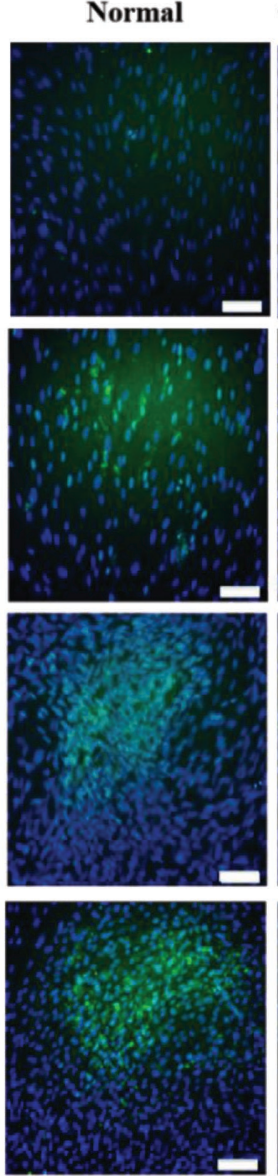

Osteoinductive
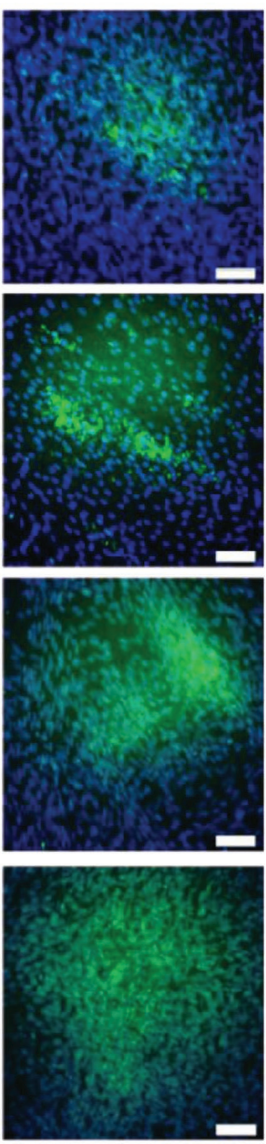

C

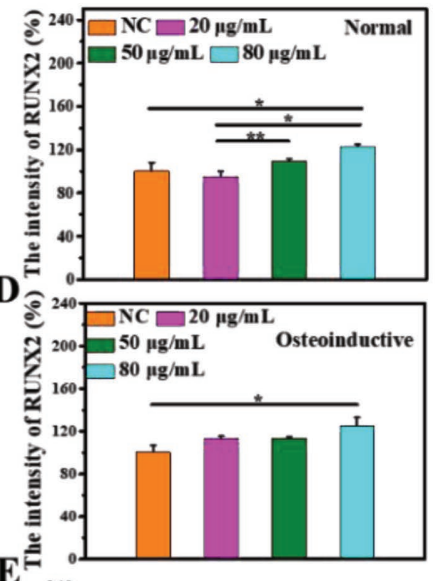

\section{$\mathbf{E}$}

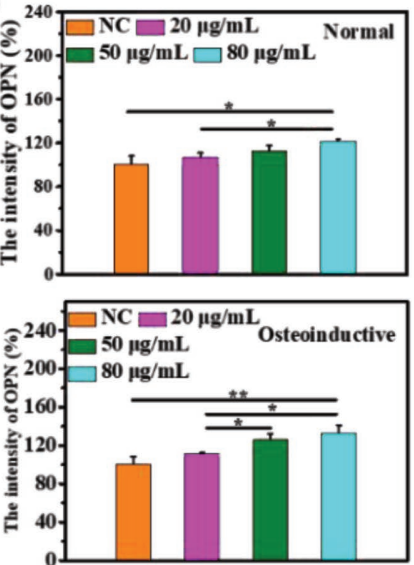

Figure 5. Immunofluorescent staining of osteogenic proteins RUNX2 and OPNin ASCs after incubated with various concentrations of BGNs in normal and osteoinductive medium for $21 \mathrm{~d}$. RUNX2 and OPN are shown as green and nuclei are stained as blue. A) Immunofluorescent staining for RUNX2 protein (scale bar = in $100 \mu \mathrm{m}$ ); B) Immunofluorescent staining of OPN protein (scale bar = in $100 \mu \mathrm{m}$ ); Relative fluorescent intensity of RUNX2 protein under normal (C) and osteoinductive medium (D); Relative fluorescent intensity of OPN protein under normal (E) and osteoinductive medium $(\mathrm{F}) . * P<0.05$ and $* * P<0.01$. Tissue culture plate $(\mathrm{NC})$ is the negative control. The relative fluorescent intensity of immunofluorescent staining was analyzed by image / based on the images.

OPN staining (green) in NC group (Figure 5A,B). However, as the addition of $\mathrm{BGNs}$, the significant positive protein staining was observed (Figure 5A,B). BGNs with $80 \mu \mathrm{g} \mathrm{mL} \mathrm{mL}^{-1}$ showed observably higher fluorescence intensity associated with RUNX2 and OPN expression as compared with other groups (Figure 5C,E). Under osteoinductive condition, the RUNX2 and OPN protein expression showed a similar tendency with that under normal condition (Figure 5D,F). The group of $80 \mu \mathrm{g} \mathrm{mL} \mathrm{m}^{-1} \mathrm{BGN}$ also showed remarkably higher fluorescence intensity associated with RUNX2 and OPN expression as compared with other groups (Figure 5). These results indicated that the appropriate concentration of BGNs could significantly enhance the osteogenic proteins expression in ASCs.

To demonstrate the effect of the uptake of BGN with different concentrations on the ASCs osteogenic differentiation at the mRNA level, we investigated the expression of osteoblastic marker genes (early-stage markers: Runx2; late-stage marker: Opn) after culture for 7 and $14 \mathrm{~d}$. All marker genes expression in all groups significantly increased from day 7 to 14 under different incubation conditions. In normal medium, as compared with other groups, the Runx2 expression on day
7 was significantly upregulated after incubation with BGNs at $80 \mu \mathrm{g} \mathrm{mL}^{-1}$ (Figure 6A). On day 14, no significant difference of Runx2 expression among various groups was observed. For Opn expression, on day 7 and 14, BGNs group with $80 \mu \mathrm{g} \mathrm{mL}$ showed the significantly high Opn level, as compared with $\mathrm{NC}$ and other BGNs groups (Figure 6B). In osteoinductive medium, on day 7 and 14, Runx2 and Opn expression in the BGNs group with optimized concentration was significantly high as compared with the NC group (Figure 6C,D). The ALP activity, Alizarin Red staining, immunofluorescence staining, and osteogenic genes analysis further demonstrated that BGNs with appropriate concentration could efficiently enhance the osteogenic differentiation of ASCs under the normal and osteoinductive conditions.

\subsection{Signal Pathway and Molecular Mechanism Investigation}

The molecular mechanisms of the osteogenic differentiation for stem cells induced by monodispersed BGNs are still not clear. It was shown that several signaling pathways such 

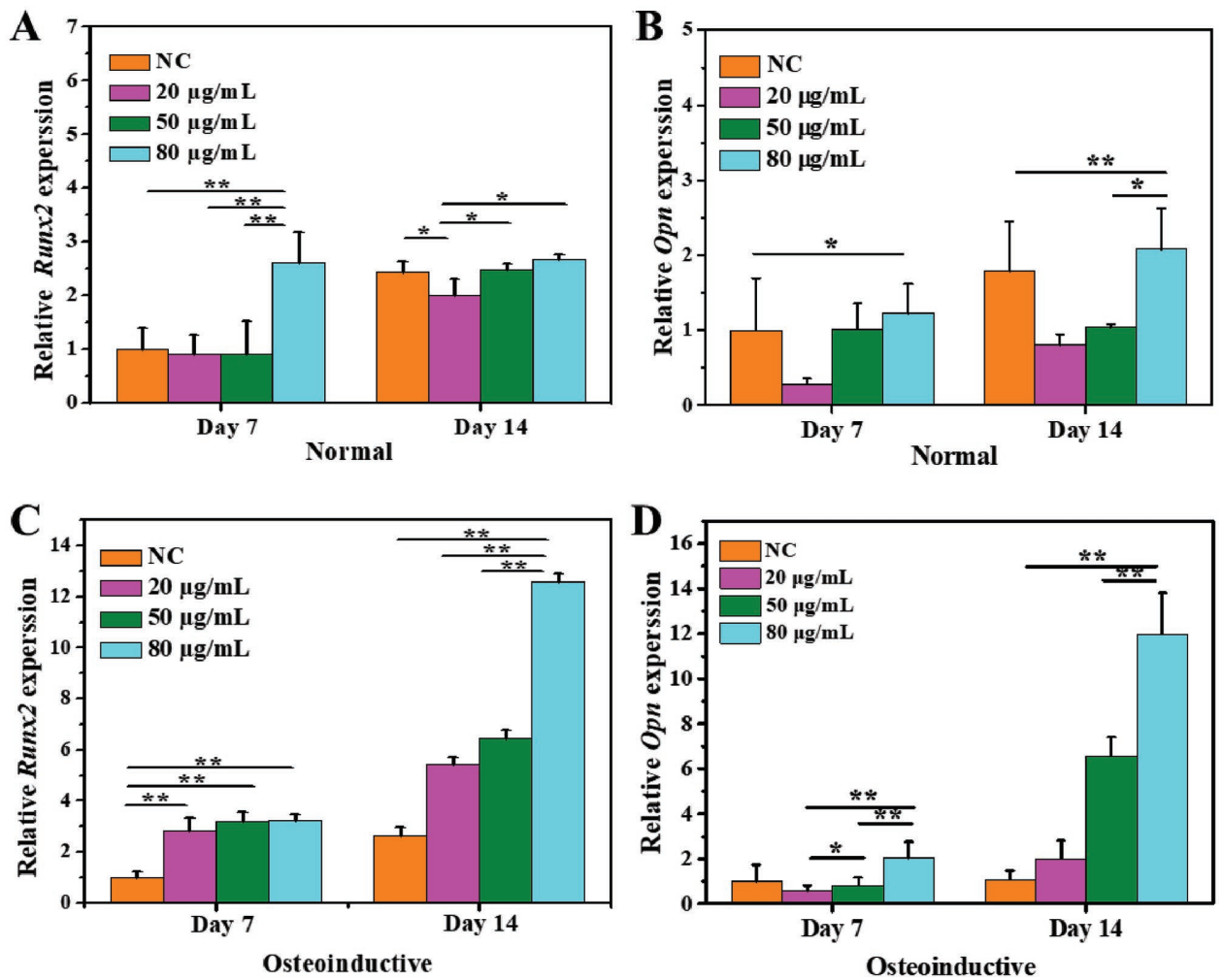

Figure 6. Relative expression of osteogenic marker genes of ASCs after cultured on normal and osteoinductive medium with the different concentration of BGNs for 7 and 14 d. Relative expression of Runx2 (A) and Opn (B) in normal medium; Relative expression of Runx2 (C) and Opn (D) in osteoinductive medium. NC is the negative control. $* P<0.05$ and $* * P<0.01$.

as BMP-2-Smads 1/5/8, TGF-beta-Smads 2/3/4, Wnt (wingless-type)-beta-catenin, MAPK/extracellular regulated protein kinases (ERK) are responsible for regulating stem cells osteogenic differentiation, and bone formation. ${ }^{[33,34]}$ Previous studies showed that bioactive micro/nanoscale surface and nanoparticles enhanced the osteogenic differentiation of MSCs through the activation of p38 MAPK, AKT/Protein Kinase B, and ERK signaling pathways. ${ }^{[35]}$ However, the TGF-beta/Smad3 signaling pathway in the osteogenic differentiation of ASCs cells induced by monodispersed BGNs was not studied. Herein, it was hypothesized that BGNs probably activated the Smad3 activity, promoted the Smad3 complex into the nucleus, and upregulated the expression of osteogenic target genes. In order to detect the hypothesis, we used inhibitor (SB 431542) to inhibit the TGF-beta type I receptors (TGF-betaR I) and then Smad3 cannot be phosphorylated, which resulted in the suppression of osteogenic marker genes. As shown in Figure 7, the uptake of BGNs significantly enhanced the expressions of osteogenic RUNX2 and OPN proteins, SMAD3, and phosphorylated SMAD3 (p-SMAD3). However, after adding inhibitor (SB 431542), the RUNX2, OPN, SMAD3, and p-SMAD3 protein levels were also decreased significantly (Figure 7A-E). Additionally, the mRNA expression levels of Runx2 and Opn were also reduced significantly, as compared with those before adding inhibitor (Figure 7F,G). These results further demonstrated that the TGF-beta/Smad3 signaling pathway plays a critical role in the effect of monodispersed BGNs on enhancing the osteogenic differentiation of ASCs. The monodispersed BGNs probably were uptaken by ASCs via endocytosis, activated the TGF-beta/Smad3 signaling pathway, enhanced the expression of osteogenic genes and proteins, and improved the osteogenic differentiation of ASCs (Scheme 1).

Previous reports have shown that monodispersed nanoparticles have different osteogenic differentiation mechanism for stem cells, as compared with the bulk materials, because nanoparticles could enter cells and may have interaction with intracellular proteins. ${ }^{[36]}$ Gold nanoparticles promote the osteogenic differentiation of BMSCs through p38 MAPK signaling pathway and human ASCs via Wnt-beta-catenin pathway. ${ }^{[2,37]}$ Iron oxide nanoparticles could also promote osteogenic differentiation of BMSCs via MAPK pathway. ${ }^{[31]}$ The silica@ $\mathrm{Ru}$ nanoparticles also showed that they could activate the Akt pathway to enhance the osteogenic differentiation of BMSCs. ${ }^{[38]}$ Nanoscale bioactive glass could improve the osteogenesis of MG-63 cells through the activation of MAPK/ERK signaling pathway. ${ }^{[29]}$ Chang group also observed that hydroxyapatite scaffolds with nanoscale structure could enhance the osteogenesis of ASCs through Akt pathway. ${ }^{[39]}$ Xiao and Wu group reported that silicate ions could enhance the osteogenic differentiation of BMSCs through the activation of Wnt pathway. ${ }^{[40]}$ In this study, we provide a new possible TGF-beta-Smads3 signaling pathway for the osteogenic differentiation of ASCs enhanced by monodispersed BGNs. TGF-beta signaling has shown that they could enhance osteogenic differentiation via the activation of MAPKs and Smad2/3 pathways. ${ }^{[41]}$ Recent studies also confirmed that the Smad pathways converge at Runx2 gene 
$\mathbf{A}$
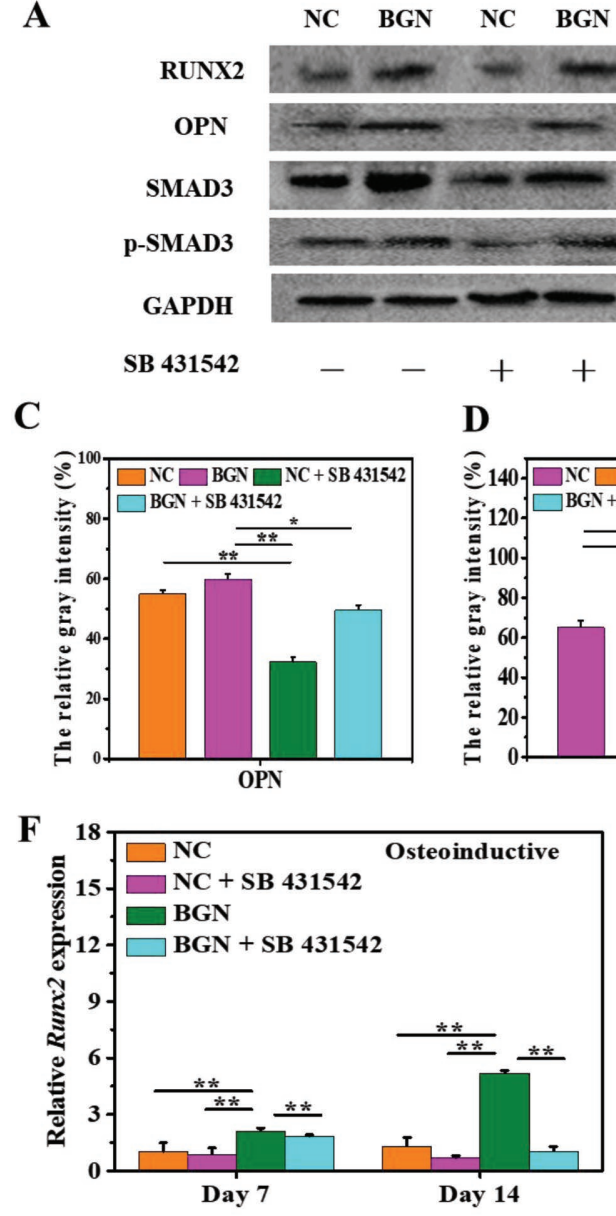

B

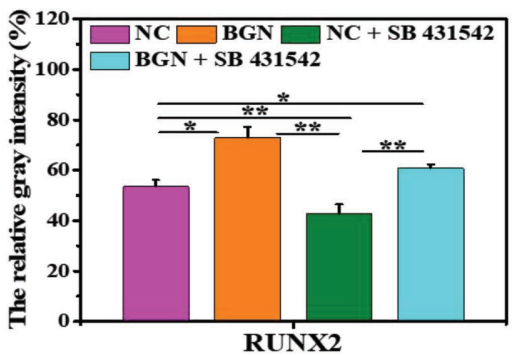

$\mathbf{E}$

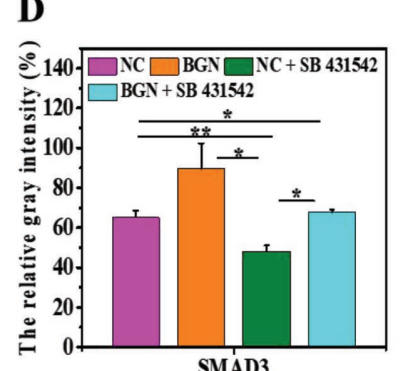

SMAD3

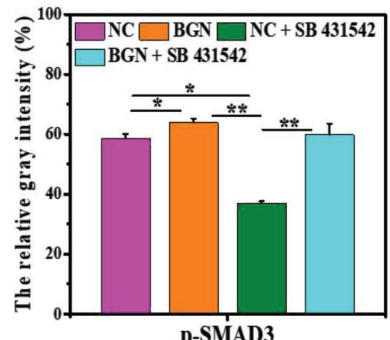

p-SMAD3

G

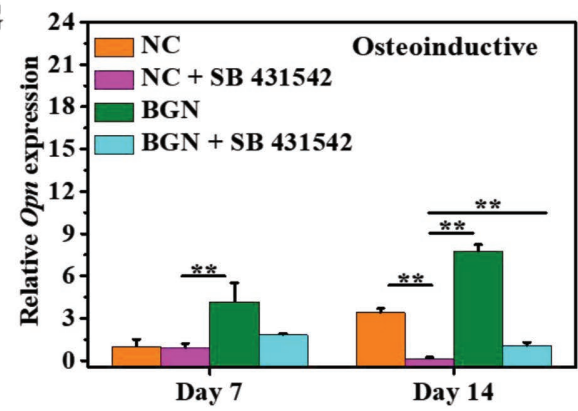

Figure 7. Osteogenic differentiation mechanism investigation of $A S C s$ induced by $B G N s\left(80 \mu g \mathrm{~mL}^{-1}\right)$ in the presence or absence of inhibitor $S B$ 431542. A) Western blotting analysis for RUNX2, OPN, SMAD3, and phosphorylated-SMAD3 (p-SMAD3) proteins expression on day 14, GAPDH is the housekeeping protein; Relative gray intensity of RUNX2 (B), OPN (C), SMAD3 (D), and p-SMAD3 (E) proteins calculated based on Western blotting patterns; Relative expression of osteogenic marker genes Runx2 (F) and Opn (G) of ASCs after culture for 7 and $14 \mathrm{~d}$. NC is the negative control. $* P<0.05$ and $* * P<0.01$

to regulate the differentiation of mesenchymal cells. ${ }^{[42]}$ Here, through inhibiting the TGF-beta type I receptor, the SMAD3, RUNX2, OPN, and p-SMAD3 protein expressions were significantly decreased. The results suggested that the addition of BGNs could efficiently activate the TGF-beta/Smad3 signaling pathway and enhance the osteogenic differentiation of ASCs. However, further in vivo study should be necessary to demonstrate the role of BGNs on regulating ASCs differentiation and bone regeneration.

\section{Conclusion}

In summary, monodispersed BGNs could be uptaken by ASCs and distributed in cytoplasm/nucleus. The internalized BGNs significantly enhanced the osteogenic differentiation of ASCs through improving their ALP activity, calcium mineralization, osteogenic proteins, and genes expressions, either normal or inductive conditions. We demonstrated that monodispersed BGNs can activate the TGF-beta signaling pathway through upregulating the level of p-SMAD3 protein, which efficiently facilitates the osteogenic differentiation of ASCs. This study suggests that monodispersed BGNs have great potential for ASCs-based bone tissue regeneration.

\section{Experimental Section}

Synthesis and Characterization of Monodispersed BGNs: The preparation process of $\mathrm{BGNs}\left(80 \mathrm{SiO}_{2}-16 \mathrm{CaO}-4 \mathrm{P}_{2} \mathrm{O}_{5}\right)$ was described in the previous study. ${ }^{[26]}$ The morphology was observed by TEM (H-8000, Hitachi). The elemental composition was measured through EDS analysis on the fieldemission scanning electron microscope (FE-SEM, SU8010, Hitachi). The FT-IR spectrometer (Nicolet 6700, Thermo Scientific Instrument) was used to analyze the chemical structure of BGNs. The spectra were obtained in the range of $4000-400 \mathrm{~cm}^{-1}$ at a resolution of $4 \mathrm{~cm}^{-1}$. The specific zeta potential and hydrodynamic size were evaluated through laser particle size analyzer (Zetasizer Nano ZS, Malvern), respectively.

Cell Culture and Cytotoxicity Analysis: ASCs were obtained from ATCC (American Type Culture Collection). The cells were cultured in (normal growth medium) Dulbecco's Modified Eagle Medium (DMEM, GIBCO) with $15 \%(\mathrm{v} / \mathrm{v})$ fetal bovine serum (FBS, Invitrogen), $100 \mathrm{U} \mathrm{\textrm {mL } ^ { - 1 }}$ penicillin and $100 \mathrm{mg} \mathrm{mL}^{-1}$ streptomycin at $37^{\circ} \mathrm{C}$ in humidified air containing $5 \% \mathrm{CO}_{2}$. The $\mathrm{BGNs}$ were dispersed in the aseptic water and 


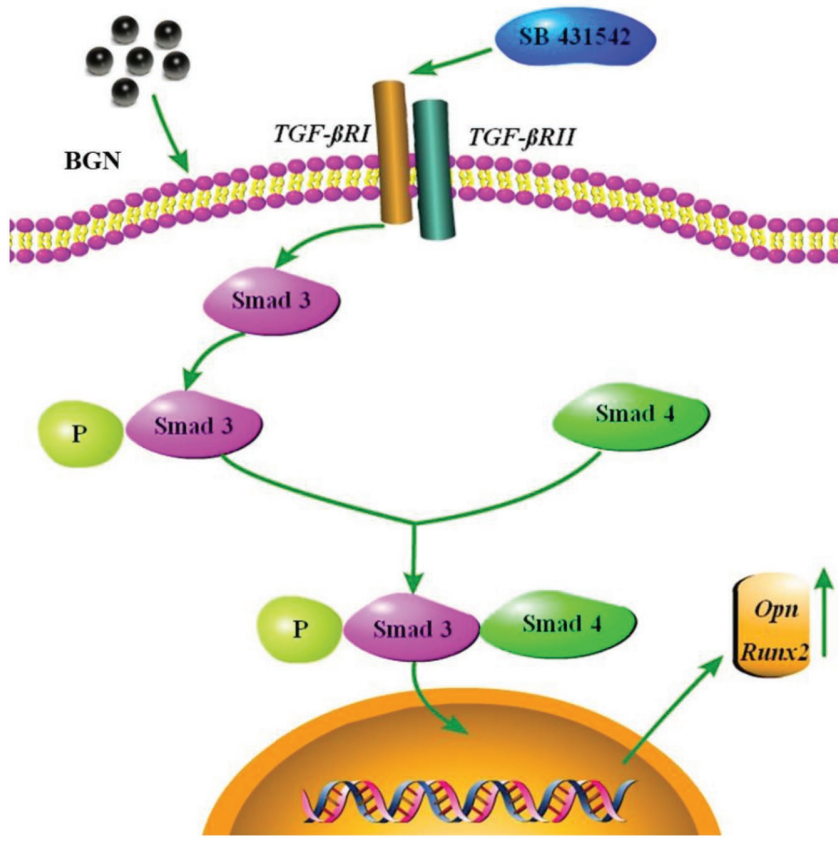

Scheme 1. Schematic illustration showing the molecular mechanism of the modulation of osteogenic differentiation of ASCs by monodispersed BGNs through TGF-beta/Smad3 signaling pathway. SB 431542 was used as a TGF-beta/Smad3 signaling pathway inhibitor.

sterilized at $60^{\circ} \mathrm{C}$ in oven overnight. The ASCs were seeded onto 96-well plates at a concentration of 1500 cells per well with normal growth medium. After $24 \mathrm{~h}$, the medium was changed to fresh growth medium with 60,120 , and $240 \mu \mathrm{g} \mathrm{mL}-1$ of BGNs. The medium was changed every $2 \mathrm{~d}$ and the cells were cultured for 1 to $5 \mathrm{~d}$. The cells on tissue culture plate in normal growth medium without BGNs were used as a negative control (NC). After 1 and $5 \mathrm{~d}$, the live/dead staining experiment was performed using a live/dead kit (Life Technologies) including the ethidium homodimer- $1\left(0.5 \times 10^{-6} \mathrm{M}\right)$ and calcein $\mathrm{AM}\left(0.25 \times 10^{-6} \mathrm{M}\right)$ (Life technologies), according to the manufacture's instruction. Then, cells were observed by an inverted fluorescence microscope (IX53, Olympus). The cell proliferation activity was analyzed by the Alamar blue assay (Molecular Probes). Briefly, after cultured for 1, 3, and $5 \mathrm{~d}$, the cells were incubated for $4 \mathrm{~h}$ in a medium containing 10\% (v/v) Alamar Blue at $37{ }^{\circ} \mathrm{C}$. The fluorescent intensity was measured at a 570/600 $\mathrm{nm}$ filter by a SpectraMax fluorescence microplate reader (Molecular Devices). The cell-free medium containing 10\% Alamar Blue was used as a blank control. At least five species per sample were tested.

Cellular Uptake Analysis of BGNs: The cellular uptake studies of BGNs in vitro were determined by a CLSM (FV1200, Olympus). To observe the fluorescence of $B G N s$, the photoluminescent $B G N s$ were prepared through zinc doping. The $\mathrm{Zn}$-doped BGNs possessed stable fluorescent properties with a green and red emission. The zinc doping did not affect the size and surface chemistry of BGNs (Table S1, Supporting Information). Briefly, after sterilized by UV light, cells were seeded on the glass cover slips with a density of 8000 cells $\mathrm{cm}^{-2}$ and incubated in the 24-well plates. After culture for $24 \mathrm{~h}$ in normal growth medium, the slips with seeded cells were incubated with medium containing fluorescent BGNs $\left(100 \mu \mathrm{g} \mathrm{mL}^{-1}\right)$ for another $24 \mathrm{~h}$. Then the glass slips were washed three times by phosphate buffered saline (PBS), fixed for $30 \mathrm{~min}$ with $4 \%$ paraformaldehyde solution, followed by the nucleus staining of 4'-6-diamidino-2-phenylindole (DAPI) for $20 \mathrm{~min}$. Finally, the cellular uptake was observed by the CLSM.

Osteogenic Differentiation Evaluations of ASCs: The osteogenic differentiation of ASCs was evaluated in normal growth medium and osteoinductive medium. The osteoinductive medium consisted of growth medium with $10 \times 10^{-9} \mathrm{M}$ dexamethasone, $10 \times 10^{-3} \mathrm{M}$

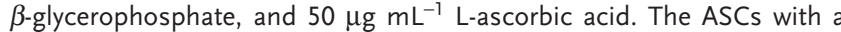
density of $2 \times 10^{4}$ cells well $^{-1}$ were seeded onto 24 -well plates and then treated with different concentrations of BGNs $\left(0,20,50\right.$, and $\left.80 \mu \mathrm{g} \mathrm{mL}^{-1}\right)$ for predetermined times. The medium was changed every 2-3 d during culture period. The osteogenic differentiation of ASCs was determined through the ALP, calcium mineralization, immunofluorescence staining, quantitative real-time polymerase chain reaction (qRT-PCR), and Western blot analysis. In order to investigate the osteogenic differentiation mechanism of ASCs induced by BGNs, the inhibitor (SB 431542) was added to the osteoinductive medium $\left(5 \times 10^{-6} \mathrm{M}\right) \cdot{ }^{[43,44]}$ The detail procedure was followed by the previous description.

ALP Activity and Calcium Deposition: The ALP activity, as an early marker of osteogenic differentiation, was assessed on days 3, 7, and 14. Briefly, at various time points, cells were rinsed with PBS solution and digested with Trypsin-EDTA (GIBCO) solution for $2 \mathrm{~min}$ at $37^{\circ} \mathrm{C}$. The cells were collected into a $1.5 \mathrm{~mL}$ tube and washed twice with PBS solution, then, adding the lysis solution to the tubes with sufficient mixing. After $10 \mathrm{~min}$ standing, the mixture was centrifuged for $15 \mathrm{~min}$ at $12000 \mathrm{rpm}$ at $4{ }^{\circ} \mathrm{C}$. Finally, the supernatant was measured for ALP activity using a Sensolyte pNPP ALP assay kit (AnaSpec, Fremont, CA, USA), and ALP activity was normalized to the total protein content measured using the Pierce BCA protein assay kit (Thermo Fisher Scientific) according to manufacturer instructions. The levels of ALP activity were determined from absorbance at $405 \mathrm{~nm}$ using a SpectraMax fluorescence microplate reader (Molecular Devices). At least three groups per sample were analyzed. The calcium deposition and extracellular-matrix mineralization of cells were tested using an alizarin red stain kit (Genmed, Quebec) according to the manufacturer instructions. In brief, the cells were cultured according to the previous description. On day 21 , the cells were washed with PBS and fixed with fixative (4\% paraformaldehyde) for $20 \mathrm{~min}$ at room temperature. Then, the cells were gently rinsed twice with PBS solution, followed by adding $200 \mu \mathrm{L}$ alizarin for $24 \mathrm{~h}$ at room temperature. After that, the cells plates were washed and the photographs were captured. The relative optical intensity for each group was calculated based on the alizarin stained pictures.

Immunofluorescence Staining: The osteogenic differentiation protein markers including RUNX2 and OPN were determined by the immunofluorescence staining. Briefly, on day 21, the cells were washed with PBS thrice and subsequently fixed with $4 \%$ paraformaldehyde for $20 \mathrm{~min}$ at room temperature. The fixed cells were washed for two times with PBS, followed by incubated with $0.1 \%$ Triton for $45 \mathrm{~min}$. After blocking with $1 \%$ bovine serum albumin for $30 \mathrm{~min}$, cells were incubated with primary antibodies RUNX2 (1:1000, Abcam, Cambridge, UK) or OPN (1:200, Abcam) overnight at $4{ }^{\circ} \mathrm{C}$. After washing three times with PBS, the appropriate secondary antibodies (Alexa 488, Thermo Fisher Scientific) were added and incubated for $1 \mathrm{~h}$ at $37^{\circ} \mathrm{C}$ in the dark. Images were captured by using a fluorescence microscope (IX53, Olympus).

Quantitative Real-Time Polymerase Chain Reaction (qRT-PCR) Assay: After 7 and $14 \mathrm{~d}$, the cells were washed twice in PBS and the ribonucleic acid (RNA) was harvested with Tripure reagent (Roche) under the instructions. Total RNA was converted to complementary deoxyribonucleic acid (cDNA) by using a reverse transcription reagent kit (Takara). The CDNA and SYBR green Master Mix were added to each well of the array plate. The qRT-PCR was performed on the fast-real-time PCR system (Applied Biosystems 7500). All the samples were first heated from room temperature to $95^{\circ} \mathrm{C}$ for $2 \mathrm{~min}$, and cycled 40 times at $95^{\circ} \mathrm{C}$ for $15 \mathrm{~s}$, then cooled down from 95 to $60^{\circ} \mathrm{C}$ for $1 \mathrm{~min}$. All target gene expression results were normalized to GAPDH. The experiment was repeated at least four times. The relative quantification was calculated by the $\Delta \Delta \mathrm{Ct}$ method.

Western Blotting Analysis: ASCs were cultured with BGNs $\left(80 \mu \mathrm{g} \mathrm{mL}^{-1}\right)$ for $14 \mathrm{~d}$ in osteoinductive medium according to the previous description. ASCs were washed three times with PBS, lysed for $30 \mathrm{~min}$ in ice-cold RIPA lysis buffer $\left(20 \times 10^{-3} \mathrm{M}\right.$ Tris. $\mathrm{HCl}, \mathrm{pH}=8.0,150 \times 10^{-3} \mathrm{M} \mathrm{NaCl}$, and $1 \%$ Triton X-100), containing protease inhibitor cocktail and phosphates inhibitor cocktail 2. After that, the mixture was centrifuged for 15 min at $12000 \mathrm{rpm}$ at $4{ }^{\circ} \mathrm{C}$. The protein concentration was measured by 
using the BCA protein assay kit (Thermo Scientific) by the supernatant. Equal aliquots of protein were heated at $100{ }^{\circ} \mathrm{C}$ for $5 \mathrm{~min}$ in $4 \times$ sample buffer (Invitrogen). And samples of the same volume were separated on $8-12 \%$ SDS-PAGE gels (Invitrogen). The protein was transferred to polyvinylidene difluoride membranes from gels, washed once with $1 \times$ TBS-T $\left(20 \times 10^{-3} \mathrm{M}\right.$ Tris- $\mathrm{HCl}, \mathrm{pH}=7.5,150 \times 10^{-3} \mathrm{M} \mathrm{NaCl}$, and $0.1 \%$ Tween), and blocked with $5 \%$ nonfat-dried milk for $40 \mathrm{~min}$ at the room temperature. The membranes were washed two times with 1 1XTBS-T followed by incubation with primary antibody of anti-SMAD3 (1:1000), phosphor-SMAD3 (1:1000), RUNX2, OPN antibodies, and GAPDH (1:1000; Abcam) as housekeeping protein overnight at $4{ }^{\circ} \mathrm{C}$. After two times wash with $1 \times$ TBS-T, the membranes were incubated with secondary antibody (Anti-mouse lgG, Abcam) for $60 \mathrm{~min}$ at the room temperature, followed by another three times wash with $1 \times$ TBS-T before protein bands visualized with ECL kit (CWBIO, Beijing, China). Finally, the membranes' pictures were taken by the chemical luminescence imaging system (Chemi Doc XRS, Bio-Rad). The relative proteins expression levels were calculated and compared by Quantity One software.

Statistical Analysis: All data were shown as mean \pm standard deviation. Statistical significance of difference between groups was assessed by a student's $t$-test. $* P<0.05, * * P<0.01$ were considered to be statistically significant.

\section{Supporting Information}

Supporting Information is available from the Wiley Online Library or from the author.

\section{Acknowledgements}

The authors acknowledge the valuable comments of potential reviewers. This work was supported by the China Postdoctoral Science Foundation (Grant No. 2017M613148), State Key Laboratory for Mechanical Behavior of Materials (No 20161801), the Natural Science Basic Research Plan in Shaanxi Province of China (Grant No. 2015JQ5165), and the National Natural Science Foundation of China (Grant No. 51502237).

\section{Conflict of Interest}

The authors declare no conflict of interest.

\section{Keywords}

adipose-derived stem cells, monodispersed bioactive glass nanoparticles, osteogenic differentiation, signaling pathway

Received: February 26, 2018 Revised: March 28, 2018 Published online: May 21, 2018

[1] A. R. Williams, J. M. Hare, Circ. Res. 2011, 109, 923.

[2] A. Uccelli, A. Laroni, M. S. Freedman, Lancet Neurol. 2011, 10, 649.

[3] N. S. Hwang, C. Zhang, Y. S. Hwang, S. Varghese, Wiley Interdiscip. Rev.: Syst. Biol. Med. 2009, 1, 97.

[4] M. Yu, B. Lei, C. B. Gao, J. Yan, P. X. Ma, Nano Res. 2017, 10, 49.

[5] M. F. Pittenger, A. M. Mackay, S. C. Beck, R. K. Jaiswal, R. Douglas, J. D. Mosca, M. A. Moorman, D. W. Simonetti, S. Craig, D. R. Marshak, Science 1999, 284, 143.

[6] H. Mizuno, M. Tobita, A. C. Uysal, Stem Cells 2012, 30, 804.
[7] M. J. Oedayrajsingh-Varma, S. M. Van Ham, M. Knippenberg, M. N. Helder, J. Klein-Nulend, T. E. Schouten, M. J. P. F. Ritt, F. J. van Milligen, Cytotherapy 2006, 8, 166.

[8] Y. X. Zhu, T. Q. Liu, K. D. Song, X. B. Fan, X. H. Ma, Z. F. Cu, Cell Biochem. Funct. 2008, 26, 664.

[9] V. Russo, C. Yu, P. Belliveau, A. Hamilton, L. E. Flynn, Stem Cell Transl. Med. 2014, 3, 206.

[10] J. Henkel, M. A. Woodruff, D. R. Epari, R. Steck, V. Glatt, I. C. Dickinson, P. F. Choong, M. A. Schuetz, D. W. Hutmacher, Bone Res. 2013, 1, 216.

[11] M. A. Woodruff, C. Lange, J. Reichert, A. Berner, F. L. Chen, P. Fratzl, J.-T. Schantz, D. W. Hutmacher, Mater. Today 2012, 15, 430.

[12] V. E. Santo, A. R. C. Duarte, E. G. Popa, M. E. Gomes, J. F. Mano, R. L. Reis, J. Controlled Release 2012, 162, 19.

[13] R. D. Gonzalez-Cruz, V. C. Fonseca, E. M. Darling, Proc. Natl. Acad. Sci. U. S. A. 2012, 109, 1523.

[14] R. Ravichandran, J. R. Venugopal, S. Sundarrajan, S. Mukherjee, S. Ramakrishna, Biomaterials 2012, 33, 846.

[15] J. M. Banks, L. C. Mozdzen, B. A. C Harley, R. C. Bailey, Biomaterials 2014, 35, 8951.

[16] F. Langenbach, J. Handschel, Stem Cell Res. Ther. 2013, 4, 117.

[17] X. J. Zhou, W. Feng, K. X. Qiu, L. Chen, W. Z. Wang, W. Nie, X. Mo, C. He, ACS Appl. Mater. Interfaces 2015, 7, 15777.

[18] B. Lei, X. F. Chen, X. Han, Z. M. Li, J. Mater. Chem. 2011, 21, 12725.

[19] B. Lei, X. F. Chen, X. Han, J. A. Zhou, J. Mater. Chem. 2012, 22, 16906.

[20] Y. M. Xue, Y. Guo, M. Yu, M. Wang, P. X. Ma, B. Lei, Adv. Healthcare Mater. 2017, 6, 1700630.

[21] S. Haimi, G. Gorianc, L. Moimas, B. Lindroos, H. Huhtala, S. Raty, K. Hannu, G. K. Sándor, C. Schmid, S. Miettinen, R. Suuronen, Acta Biomater. 2009, 5, 3122.

[22] S. Dobbenga, L. E. Fratila-Apachitei, A. A. Zadpoor, Acta Biomater. 2016, 46, 3.

[23] Y. Z. Du, J. Ge, Y. N. Li, P. X. Ma, B. Lei, Biomaterials 2018, 157, 40.

[24] C. Q. Yi, D. D. Liu, C. C. Fong, J. C. Zhang, M. S. Yang, ACS Nano 2010, 4, 6439.

[25] J. Isaac, J. Nohra, J. Lao, E. Jallot, J. M. Nedelec, A. Berdal, J. M. Sautier, Eur. Cells Mater. 2011, 21, 130.

[26] Y. M. Xue, Y. Z. Du, J. Yan, Z. Q. Liu, P. X. Ma, X. F. Chen, B. Lei, J. Mater. Chem. B 2015, 3, 3831.

[27] M. Yu, Y. M. Xue, P. X. Ma, C. Mao, B. Lei, ACS Appl. Mater. Interfaces 2017, 9, 8460.

[28] W. Y. Gong, Y. M. Dong, X. F. Chen, B. Karabucak, Chin. J. Dent. Res. 2012, 15, 145.

[29] W. Y. Gong, Y. M. Dong, S. N. Wang, X. J. Gao, X. F. Chen, RSC Adv. 2017, 7, 13760.

[30] X. Guo, J. Liao, H. Park, A. Saraf, R. M. Raphael, Y. Tabata, F. K. Kasper, A.G. Mikos, Acta Biomater. 2010, 6, 2920.

[31] Q. W. Wang, B. Chen, M. Cao, J. F. Sun, H. Wu, P. Zhao, J. Xing, Y. Yang, X. Q. Zhang, M. Ji, N. Gu, Biomaterials 2016, 86, 11.

[32] J. C. Li, Y. Chen, Y. J. Yang, N. Kawazoe, G. P. Chen, J. Mater. Chem. B 2017, 5, 1353 .

[33] F. Ng, S. Boucher, S. Koh, K. S. R. Sastry, L. Chase, U. Lakshmipathy, C. Choong, Y. Zheng, M. C. Vemuri, M. S. Rao, V. Tanavde, Blood 2008, 112, 295.

[34] X. Su, L. Liao, Y. Shuai, H. Jing, S. Liu, H. Zhou, Y. Liu, Y. Jin, Cell Death Dis. 2015, 6, 221.

[35] K. Jiao, L. N. Niu, Q. H. Li, F. M. Chen, W. Zhao, J. J. Li, J. H. Chen, C. W. Cutler, D. H. Pashley, F. R. Tay, Acta Biomater. 2015, 19, 23.

[36] K. N. Yang, W. P. Cao, X. H. Hao, X. Xue, J. Zhao, J. Liu, Y. L. Zhao, J. Meng, B. Y. Sun, J. C. Zhang, X. J. Liang, Nanoscale 2013, 3, 1205.

[37] S. Y. Choi, M. S. Song, P. D. Ryu, A. T. N. Lam, S. W. Joo, S. Y. Lee, Int. J. Nanomed. 2015, 10, 4383. 
[38] Y. Liu, N. Huang, Y. F. Yu, C. P. Zheng, N. Deng, J. Liu, J. Mater. Chem. B 2016, 4, 4389.

[39] L. G. Xia, K. L. Lin, X. Q. Jiang, B. Fang, Y. J. Xu, J. Q. Liu, D. L. Zeng, M. L. Zhang, X. L. Zhang, J. Chang, Z. Y. Zhang, Biomaterials 2014, 35, 8514 .

[40] P. P. Han, C. T. Wu, Y. Xiao, Biomater. Sci. 2013, 1, 379.
[41] D. Y. Rhyu, Y. Q. Yang, H. J. Ha, G. T. Lee, J. S. Song, S. T. Uh, H. B. Lee, J. Am. Soc. Nephrol. 2005, 16, 667.

[42] K. S. Lee, S. H. Hong, S. C. Bae, Oncogene 2002, 21, 7156.

[43] N. Quarto, S. L. Li, A. Renda, M. T. Longaker, Stem Cells 2012, 30, 2709.

[44] K. Koli, M. J. Ryynanen, J. Keski-Oja, Bone 2008, 43, 679. 